\title{
tic\&société
}

Vol. 10, $N^{\circ} 1 \mid 1$ er semestre 2016

Contrôle social, surveillance et dispositifs numériques

\section{Négocier (avec) la surveillance électronique}

Johann Chaulet and Camille Allaria

\section{(2) OpenEdition}

\section{Journals}

Electronic version

URL: http://journals.openedition.org/ticetsociete/2007

DOI: 10.4000/ticetsociete.2007

\section{Publisher}

Association ARTIC

Electronic reference

Johann Chaulet and Camille Allaria, « Négocier (avec) la surveillance électronique », tic\&société

[Online], Vol. 10, N 1 | 1er semestre 2016, Online since 06 November 2016, connection on 19 April

2019. URL : http://journals.openedition.org/ticetsociete/2007 ; DOI : 10.4000/ticetsociete.2007

This text was automatically generated on 19 April 2019.

Licence Creative Commons 


\title{
Négocier (avec) la surveillance électronique
}

\author{
Johann Chaulet and Camille Allaria
}

\section{Introduction}

1 Les technologies de saisie à distance d'informations sur les personnes se démocratisent et se multiplient, s'invitant dans des sphères sociales de plus en plus nombreuses. Qu'elles s'intègrent dans des relations hiérarchisées ou impliquant des formes de subordination ${ }^{1}$ ou qu'elles se déploient au sein de relations horizontales, entre pairs (Chaulet, 2007 ; Green, 2002), ces techniques équipent les jugements qui reposent pour partie sur la distance entre un comportement saisi techniquement et un comportement attendu. Nous proposons d'étudier ici la façon dont la conformité des comportements est négociée au sein de ces dispositifs de surveillance qui s'appuient, dans une large mesure, sur des technologies numériques de saisie des activités individuelles. Nous mobiliserons pour ce faire les données de deux enquêtes ethnographiques : la première portant sur le dispositif d'assignation à domicile sous surveillance des condamnés, mis en place au moyen du bracelet électronique; la seconde sur le travail fortement équipé des salariés en centre d'appels téléphoniques. Après avoir rapidement présenté ces dispositifs, nous analyserons les formes de négociation et de contournement, tant techniques que sociales, auxquelles leur fonctionnement donne lieu. En conclusion, l'examen de ces formes de négociation nous mènera à interroger la portée critique ou subversive de ces processus opposés aux technologies de pouvoir et de gouvernement (Foucault, 1975).

\section{Les deux terrains d'enquête}

\section{La surveillance des salariés en centre d'appels}

2 Les observations réalisées en centre d'appels portent sur des opérations de vente de produits d'assurance (décès et invalidité). L'ensemble des communications téléphoniques 
transite par un système informatique qui assure la numérotation depuis des fiches informatisées et produit nombre d'informations stockées à leur tour au sein du réseau (durée et nombre des communications, appels "argumentés», temps de pause, etc.). Ainsi sont enregistrées des données individualisées relatives au nombre de communications établies par les téléacteurs, à leur durée, au nombre de vente réalisées, etc. Certaines de ces informations sont par ailleurs reprises et rendues visibles de tous sur des panneaux d'affichage situés à l'intersection de plusieurs "rosaces", des postes de travail collectifs où sont installés les téléacteurs. En outre, l'équipement permet que les superviseurs, munis de casques, écoutent les conversations en cours. Les cinq superviseurs qui contrôlent le plateau sont debout; ils peuvent ainsi plus aisément observer les trois groupes de six téléacteurs qu'ils ont la charge de surveiller. Les centres d'appels offrent donc à voir un dispositif panoptique (Foucault, 1975) dans la mesure où la surveillance peut être totale et complète et échapper, parfois, à la connaissance de ceux qui ne savent jamais exactement quand et comment ils sont surveillés (Chaulet, 2006).

Deux semaines d'observation ont été effectuées en centre d'appels, permettant à l'enquêteur de se familiariser avec l'activité et son fonctionnement et de réaliser un certain nombre d'entretiens informels complétés ensuite par la réalisation d'entretiens semi-directifs auprès de téléopérateurs (du centre d'appels où les observations ont été réalisées ainsi que d'autres entreprises).

\section{La surveillance électronique des condamnés}

4 Le placement sous surveillance électronique est une mesure d'aménagement permettant d'exécuter une peine d'emprisonnement à son domicile. Il peut également être utilisé comme une alternative à la détention provisoire ou dans le cadre d'une surveillance électronique de fin de peine. En fonction de la situation - familiale, professionnelle, sanitaire - individuelle, le juge établit les horaires auxquels le condamné doit être chez lui et ceux auxquels il doit/peut être en dehors de son domicile. Le matériel de surveillance électronique renvoie automatiquement les informations au pôle de surveillance relatives à la présence ou à l'absence du condamné. En cas d'alarme signalant un incident, le surveillant avertit le juge et le conseiller pénitentiaire d'insertion et de probation (CPIP) du condamné. Il téléphone également au condamné qui doit justifier son retard ou son absence par téléphone, puis faxer les documents permettant de justifier ses dires.

5 Un temps d'enquête plus long a été dédié au placement sous surveillance électronique (PSE) pour la raison principale qu'il s'agissait d'une recherche de doctorat. Les mois de septembre et d'octobre 2009, de février 2010 et d'avril 2011 ont été consacrés à l'ethnographie du pôle de surveillance électronique du centre pénitentiaire des Baumettes, à Marseille. Des entretiens exploratoires ont été menés avec les agents de surveillance du pôle centralisateur. Sur des plages de deux heures, de façon quotidienne, les appels téléphoniques des surveillants ont été enregistrés, avec leur accord. À ces enregistrements sont associées des notes relatant les interactions des surveillants au moment de leur appel. Au total, 251 appels ont été enregistrés et retranscrits. Entre août 2010 et mars 2011, l'enquête a été menée principalement au sein des services pénitentiaires d'insertion et probation (SPIP) de Marseille, Toulon, Nice et Aix-enProvence auprès de juges d'application des peines et de condamnés sous surveillance électronique. 


\section{Les caractéristiques communes de ces deux dispositifs de surveillance}

6 L'un des intérêts de notre analyse réside dans sa dimension comparative. Il est donc nécessaire de procéder à une mise au point des traits communs à nos deux terrains d'investigation. De la même manière, leurs différences - tant du point de vue pratique qu'organisationnel - nous permettront de comprendre ce qui relève de contingences spécifiques ou ce qui peut, au contraire, être généralisé à l'ensemble des situations analogues.

7 Dans l'un et l'autre cas, les comportements individuels sont fortement cadrés par des règles précises donnant lieu à des " programmes d'action » (Akrich, 1990) stricts, intégrés au fonctionnement même des machines (Chaulet, 2006, 2007) et dont le respect est contrôlé par des surveillants équipés de dispositifs de qualification, complets et complexes (Soler et Trompette, 2010). Ils sont conçus pour capturer de façon automatique l'« activité en train de se faire » des utilisateurs, elle-même rapportée à une " grammaire d'action" (Agre, 1994), préalablement établie par une autorité institutionnelle supérieure. Ces dispositifs peuvent être qualifiés de "méfiants", dans la mesure où ils sont conçus pour réagir à l'écart négatif avant de valoriser des comportements « positifs ». Comme le note Jaeger (2002, p. 84) :

« ce que mesurent les systèmes d'information comme les divers autres indicateurs de performance mis en place, ce n'est pas la valeur créée par le travail des téléopérateurs, mais le degré de conformité de leurs pratiques aux prescriptions de l'entreprise »

8 ou, plus largement, de l'organisation à laquelle ils sont soumis. Dans le cas du PSE, il ne s'agit pas non plus, pour le dispositif technique, de mesurer la production de valeur (comme le degré d'intérêt d'une activité justifiant un retard), mais de mesurer un écart à la norme prescrite par le juge d'application des peines (JAP).

9 L'étude de deux situations de surveillance distinctes montre également qu'il n'existe pas de définition univoque de ce à quoi renvoie la conformité. Une zone d'incertitude demeure, qui laisse place à l'interprétation et à la mise en œuvre de définitions personnelles de ce vers quoi doit tendre l'activité (de travail ou de liberté contrôlée). Ces jugements relatifs, qui mettent en jeu les visions du monde et les principes normatifs et moraux individuels de ceux qui les exercent, préexistent à la mise en place du dispositif technique et se sont construits, au moins dans une certaine mesure, indépendamment de lui.

10 Les formes de jugement et de qualification de la conformité et les formats d'enquête des surveillants mériteraient des développements supplémentaires ; notre analyse se bornera ici à la question de la négociation à laquelle ces jugements donnent lieu. Afin de saisir le cadre au sein duquel s'insèrent ces formes de négociation, nous examinerons pour commencer les manipulations techniques et les modes de production de conformité, effective ou simulée. Il s'agira enfin d'interroger, pour conclure, la portée subversive des contournements et des négociations étudiées. 


\section{Jouer avec les interstices normatifs des dispositifs de surveillance}

11 Nous nous intéresserons ici aux techniques développées par les surveillés pour éviter d'éveiller les soupçons du surveillant - et par là même l'inviter à initier une enquête. Il convient d'introduire la notion d' "indices de conformité » - ou de non-conformité -, éléments qui sont de nature à faire "pencher » le surveillant dans un sens ou dans un autre. Comme le note Marie-Anne Dujarier, «lorsqu'ils ne peuvent échapper [à l'évaluation], car elle comporte des enjeux juridiques, financiers ou économiques, les travailleurs simulent au mieux les résultats attendus et dissimulent ce qu'ils font vraiment » $(2010$, p. 146). L'enjeu n'est alors plus tant d'être conforme que de produire toujours les indices qui invitent le surveillant à penser qu'on l'est. Nous allons voir les enjeux pratiques que revêtent ces indices et comment il est en effet possible en situation de rendre visibles les comportements légitimes et invisibles les comportements déviants.

Bien que nous nous attachions à étudier leurs contournements, dans la plupart des cas

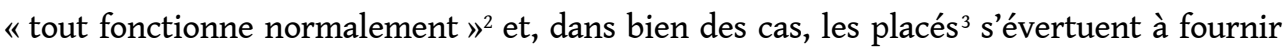
aux surveillants les indices de leur conformité effective. De fait, les dispositifs de surveillance "panoptique » produisent des effets, largement démontrés, de police des comportements (Foucault, 1975; Lianos, 2003). En centre d'appels, les téléopérateurs s'assurent régulièrement que les ventes qu'ils réalisent sont bien prises en compte et enregistrées dans les statistiques par les superviseurs. Ils développent même, dans certains cas, des logiques de vérification, tel ce placé qui téléphone au pôle de surveillance pour s'assurer que son boîtier fonctionne correctement (i.e. qu'il est effectivement surveillé) ou que son nouveau contrat de travail a bien été faxé. Cet « individu préoccupé » s'approprie le système de collecte d'information et fait un pas vers la maîtrise des données le concernant.

\section{Démystifier le matériel de surveillance}

13 Ces situations équipées offrent aux surveillés des occasions d'accroitre leur expertise technique (en étant très régulièrement aux prises avec le fonctionnement concret $\mathrm{du}$ dispositif de surveillance et en interaction avec les surveillants et/ou d'autres surveillés). Ils sont alors ensuite en mesure de mobiliser l'expertise acquise, individuellement et collectivement, pour s'approprier le dispositif et, éventuellement, le contourner. Dans le cas de la surveillance des prisonniers, les imperfections techniques des nombreux matériels impliqués dans le dispositif (bracelet, connexion Internet personnelle des placés, boîtier, etc.) obligent les surveillants à assurer par téléphone une assistance technique régulière auprès des placés. De même, tout au long du placement, les surveillants vont solliciter à distance les prisonniers pour résoudre, depuis leur domicile, les pannes et autres incidents techniques. Si ce dispositif peut initialement apparaitre comme le symbole tout-puissant de l'institution pénale, l'expérience, concrète et répétée, de ses failles conduit à sa désacralisation. L'argument technique peut devenir un élément phare dans la justification que le placé avance lorsque le surveillant constate une alarme (nous reviendrons sur cet élément dans la troisième partie).

« Le téléphone sonne tout seul. Vous avez une panne électrique ? Une coupure d'alimentation, une défaillance de ligne téléphonique à 14 heures. Si je vous le dis. Mais 
c'est ponctuel. C'est peut-être le simple fait d'utiliser le téléphone au mauvais moment » (conversation enregistrée au pôle de surveillance électronique des Baumettes le 27 octobre 2009).

Sans toujours prendre le risque de tester volontairement le dispositif, les surveillés sont amenés à développer des connaissances, à constater des régularités qui équipent leurs façons de faire et de se comporter. Ainsi, cet homme placé sous bracelet électronique constate que, lorsqu'il s'absente pour boire un verre avec des amis, son absence est détectée, quand bien même le bar se situe près de chez lui. En revanche, aucune réaction ne se produit lorsqu'il sort acheter du pain en dehors des heures de sortie prévues ${ }^{4}$. Ces constats répétés lui permettent de déduire que le dispositif est faillible, que l'erreur soit attribuée à la machine ou à celui qui la manipule au sein du pôle de surveillance.

«Surtout qu'il faut savoir que la machine elle a un écart de sensibilité de 10 minutes. Donc, celui qui va avoir 13 minutes de retard, à 3 minutes près, il n'avait pas de... par rapport aux événements du logiciel, puisque l'écart de sensibilité est de 10 minutes. Celui qui va arriver avec 9 minutes de retard il va même pas nous apparaître. Celui qui va arriver avec 11 minutes de retard il va apparaître. Alors, on va dire qu'à 2 minutes près, c'est pas de bol pour lui. À ce niveau-là, on va dire qu'on ne va pas être strict. Un peu moins strict qu'au centre de semi-liberté, bien qu'ils aient aussi une marge de manœuvre. En général, ils gèrent à partir de 15 minutes. Au centre de semi-liberté, pas tous mais, bon, beaucoup. On va dire qu'on a une tolérance par rapport à ça » (agent PSE, entretien $n$ $\left.{ }^{\circ} 2\right)$.

On peut noter que la situation de surveillance concrète et physique (le centre de semiliberté) fait office de référence : elle est celle à laquelle est comparée la situation de surveillance équipée. Elle est d'ailleurs, tel que le dit le surveillant, largement comparable à celle-ci dans la marge de manœuvre qu'elle autorise. Pourtant, on peut noter une différence non négligeable : si cette marge relève d'une largesse, ou tout du moins d'une latitude volontairement permise par le surveillant, dans le cas de surveillance non équipée, elle acquiert, lorsque le dispositif médiatise cette relation, un caractère systématique, déconnecté de toute volonté du surveillant. C'est la machine qui accorde 10 minutes de grâce et non plus le surveillant.

\section{La manipulation experte des machines et le détournement de la traçabilité médiatique}

En centre d'appels, la traçabilité est essentiellement assurée par l'outil informatique; certaines étapes nécessitent malgré tout l'intervention des téléacteurs, qui peuvent alors agir sur les données saisies afin d'éviter la mobilisation ultérieure de traces qui pourraient leur être préjudiciables. Ainsi, nous constatons que de nombreux fichiers audio ne sont pas signés par les téléacteurs malgré les rappels réguliers de leurs superviseurs. Ces pratiques rendent plus difficile l'attribution des fautes éventuellement constatées plus tard sur lesdits enregistrements. À l'inverse, dans des situations délicates où l'activité peut être remise en question, il est important de pouvoir mobiliser la machine pour «montrer patte blanche", pour s'assurer que le surveillant a conscience que le surveillé a fait comme il fallait ou, s'il ne le pouvait pas, aussi bien qu'il était en mesure de le faire. Dans l'extrait qui suit, Divy nous explique comment il (se) joue des différents indices produits par le système. Cet exemple constitue l'un des nombreux exemples de stratégies analogues développées par les surveillés aguerris. 
" Toi, de ton côté, si tu veux avoir ces résultats et être tranquille, il n'y a pas 36 solutions. Ce que tu fais... Moi, comme je sais que j'arrive à vendre assez facilement, je me dis : « Je n'ai pas besoin de téléphoner aux sept mecs, pour avoir six refus et une vente. Si je ne téléphone qu'à trois gars, ça ira, je sais qu'en trois coups, je ferai la vente, il n'y a pas de problème. Par contre, si je fais ça, ça va me faire quoi ? Ça va me faire deux refus et un accord. Donc, ça ne va pas être bon sur mes contacts 5 . Donc, là, ils vont venir me voir et me dire : «Attends, t'appelles pas assez. Qu'est-ce que tu fais ? C'est pas bon ».

Donc, moi, ce que je fais, c'est que, euh... Si tu veux, tu peux être en écoute que si t'es en train de parler effectivement au téléphone. Donc, moi, ce que je fais, c'est que je fais tourner des fiches et, au bout de 1 minute de chaque fiche, je mets « Ne répond pas », tac. Pendant tout ce temps où je mets " Ne répond pas ", eux, les superviseurs, sur l'ordinateur, ils voient un appel, une minute, ça ne répond pas. C'est normal! « Une minute, ça ne répond pas, il a essayé 1 minute et ça ne répond pas... »

J.C. : Et il se passe quoi, en fait?

Divy : Ben, je t'explique. C'est-à-dire que, eux, pendant qu'ils surveillent, moi, pendant ce temps-là, je ne fais rien c'est-à-dire que j'ouvre la fiche et j'éteins le combiné, si tu veux. J. C. : Donc, ça ne sonne pas.

Divy : Voilà, donc ça ne sonne pas, donc, moi, je suis tranquille, j'ai aucune chance que la personne décroche » (Divy, téléacteur).

On note que déjouer la dynamique de contrôle requiert, certes, un certain savoir-faire technique mais également d'autres types de compétences. En effet, Divy ne peut manipuler les indicateurs (nombre de contacts par heure) que parce qu'il se sait suffisamment compétent pour atteindre les objectifs qui lui sont fixés. Cinq appels dans l'heure lui suffiront à réaliser deux ventes ; il est donc inutile qu'il effectue quinze appels et peut « tricher » sur ce point. La connaissance précise de la règle est indispensable à son contournement. Par ailleurs, son expérience du système lui permet de savoir que les écoutes ne peuvent être réalisées que lorsqu'une conversation a effectivement été entamée. Enfin, il connaît suffisamment bien les techniques d'enquête des superviseurs pour pouvoir simuler une situation "normale » et produire les indices de sa conformité, alors même que son comportement «dévie ». Comme il le dit, «ils voient un appel, 1 minute, ça ne répond pas. C'est normal ». Rien dans son comportement ne " dépasse ». Il ne fait réagir ni le surveillant ni le dispositif technique chargé de le surveiller. Aucune alarme ne vient initier d'enquête approfondie sur son comportement. Son contournement est pourtant très relatif dans la mesure où, à l'échelle de la journée, son activité ne s'écarte pas du cadre prescrit. C'est un bon vendeur et il atteint tous les jours les objectifs fixés par la direction. D'un point de vue organisationnel, on peut donc imaginer que son comportement pourrait être toléré quand bien même il serait repéré par ses supérieurs.

18 Si nous ne pouvons ici prendre le temps d'en faire la démonstration, nous constatons pourtant que l'évaluation de la conformité dans un contexte fortement technicisé laisse fréquemment réapparaître le corps (de façon médiatisée, lors des appels téléphoniques entre placé et surveillant par exemple, ou non médiatisée par l'observation directe des téléopérateurs installés à leur poste de travail). De la même façon, certains contournements des dispositifs de saisie sont précisément conditionnés par l'absence des corps. Max peut ainsi doubler son temps de pause sans prendre de risque parce qu'aucun superviseur n'est présent pour constater qu'il n'a pas cliqué sur le bouton déclenchant le décompte de son temps de repos (et qu'aucun compteur ne défile donc, en grands chiffres 
rouges, sur son écran) avant de quitter son poste de travail. De fait, nous constatons que si les machines continuent à enregistrer et stocker les agissements des téléacteurs, l'absence du superviseur sur le plateau autorise de nombreux contournements que sa présence rend impossibles. De même, bien des manipulations effectuées par les prisonniers pour disparaître ne sont possibles que parce la surveillance s'effectue intégralement à distance et qu'aucun agent pénitentiaire n'est physiquement présent pour les regarder faire.

Nos deux terrains se distinguent ici dans les formes de manipulation qu'ils donnent à observer. En effet, les porteurs de bracelets électroniques flirtent avec les limites techniques du dispositif de surveillance quand les salariés des centres d'appels manipulent plus activement les machines qui saisissent et enregistrent leur activité. Pourtant, l'un et l'autre de ces processus procèdent d'une logique comparable de jeu avec les marges et d'utilisation des limites du système. On peut alors avancer l'idée d'une « carrière du surveillé ", processus au cours duquel celui-ci va peu à peu développer ses connaissances et son expertise du dispositif de contrôle. Passé le premier temps de l'inquiétude concernant un matériel que l'on pense tout-puissant, celui qui fait l'objet du contrôle va s'autoriser un jeu avec le matériel lui permettant de percevoir les premières limites de la saisie. S'ensuit alors une éventuelle phase d'expertise où il saura faire parler la machine en sa faveur, voire brouiller les pistes pour disparaître et s'assurer de toujours produire les indices de sa conformité, effective ou non. Comme nous allons le voir dans la partie qui suit, cette expertise ne se limite pas à la dimension technique mais concerne également les dimensions sociales de la négociation.

\section{Comment redevenir conforme}

Outre le face-à-face avec la machine, la négociation se développe également au sein des interactions et dans les relations qu'établissent surveillants et surveillés. Quand bien même nos exemples offrent à voir des formes de saisie fortement instrumentées, elles laissent souvent, en dernière analyse, une place à l'échange humain puisque c'est aux surveillants que les surveillés doivent rendre des comptes concernant des comportements que la machine saisit, met en lumière parfois, mais ne sanctionne pas ${ }^{6}$. Si la saisie est automatique, la sanction, elle, ne l'est pas. Pour tenter de mettre en ordre ces différentes façons de négocier avec l'humain, nous traiterons ici des registres de justification que mobilisent les surveillés.

\section{Les registres de justification}

21 La résistance peut tout d'abord se placer sur le registre de la justification a posteriori de la déviance constatée. Il s'agit, dans ce cas, de fournir à celui qui réclame des explications, de «bonnes raisons » d'avoir failli aux impératifs fixés. La négociation n'est ni purement matérielle ni totalement discursive; elle fait au contraire appel, nous allons le voir, à différents « régimes d'engagement », naviguant pour ce faire sans cesse entre les régimes du proche, du plan ou de la justice (Thévenot, 2006).

Parmi les différentes façons dont les surveillés se justifient seront développés ici : la mobilisation du registre technique, celle des grandeurs et enfin la contre-enquête que les surveillés opposent à l'enquête des surveillants. 


\section{Le registre technique}

les téléacteurs déclarent que «l'enregistrement a buggé » quand aucun fichier n'accompagne l'accord du client qu'ils n'ont, en réalité, pas obtenu. Les objectifs précis de production peuvent être difficiles à atteindre; la qualité des fichiers transmis aux téléacteurs peut alors être mise en cause par ces derniers pour expliquer leur faible performance. Ces derniers se plaignent fréquemment de la présence dans les fichiers de prospects ayant déjà, à plusieurs reprises, répondu par la négative aux sollicitations de leurs collègues et qui devraient logiquement être éliminés du fichier puisqu'il est certain qu'ils ne seront jamais sensibles à leurs arguments. La présence de ces fiches " parasites » explique pourtant en partie les taux d'accords (TA) insuffisants, l'écart à la norme qui leur est reproché.

«En fait, la performance de production de rendez-vous va dépendre de deux éléments : les TA, premier élément... On va dire que ça détermine $60 \%$ de la prod ou $70 \%$ même, les TA, c'est-à-dire leur envie de travailler... Ça, ça détermine $70 \%$ de la prod selon que ton $\mathrm{TA}$, il est bien ou il n'est pas bien mentalement, tu vois. [...] Et après, les $30 \%$ qui restent ou les $20 \%$ qui restent peut-être, ce sont les fichiers, les fichiers informatiques parce que, évidemment, si on te donne des fichiers où les gens ont déjà été appelés 10 fois et tu les rappelles toi par-dessus, évidemment que tu vas te faire jeter à $98 \%$ ou $99 \%$. Tandis que si on te donne un fichier où les gens ont été appelés zéro fois ou une fois, là, forcément, t'as beaucoup plus de chances d'aboutir » (Mathieu, téléacteur).

Puisque ces conditions déterminent leur propre activité indépendamment de leur comportement, il s'agit pour les téléacteurs de parvenir à exhiber les éléments qui concourent à fournir la preuve de la mauvaise qualité des outils qui leur sont fournis. La technique et sa faillibilité avérée sont mobilisées comme alibi, à tort ou à raison. Ainsi, ce placé qui veut participer à une soirée dansante près de chez lui coupe le courant dans son logement et prétexte une panne d'électricité pour expliquer le dysfonctionnement repéré par l'institution. Ce type de justification opéré par les surveillés constituerait une première forme de critique de la légitimité du surveillant qui, dans le système et l'agencement sociotechnique de ces formes de surveillance, apparaît, nous l'avons vu plus tôt, comme le garant de la technique et qui mobilise pourtant des instruments de saisie faillibles, qui n'enregistrent qu'une réalité incomplète voire biaisée.

\section{Les grandeurs}

Dans les contextes de surveillance équipée également, nous constatons que «tout en réduisant l'incertitude quant au comportement des subordonnés, la règle en crée donc une autre qui tient à la question de savoir jusqu'à quel point ces derniers choisiront de l'utiliser comme une protection contre l'arbitraire du supérieur » (Crozier et Friedberg, 1992, p. 90). En effet, la justification fait fréquemment appel à des principes de justice supérieurs (Boltanski et Thévenot, 1991), qui soutiennent les règles auxquelles sont soumis les surveillés. Tous n'ont cependant pas le même droit de cité dans les arènes constituées lors des procès en justification. Les différents registres de justification pèsent différemment sur les jugements des surveillants et l'attribution des " grandeurs ». Dans le cas du bracelet électronique, invoquer la cité «industrielle » semble par exemple plus pertinent et opérant que le fait d'invoquer la cité «domestique»; quand il s'agit de

tic\&société, Vol. 10, Nㅜ 1 | 1 er semestre 2016 
paraître « grand » aux yeux du surveillant, la hiérarchie se fait au profit du travail compte tenu des objectifs généraux que se fixe le dispositif, ainsi que la conception du «bien » (pour les placés) qui correspond.

«Il m'appelle pour un changement d'horaire ; il me le demande par téléphone. Alors, je lui ai expliqué, déjà, qu'on ne pouvait pas faire ça par téléphone et que, de toute manière, il fallait que j'évalue sa situation. Et alors, lui, il avait besoin de ce changement d'horaire, donc il continuait à me parler : "Oui, je ne peux pas aller voir ma mère à l'hôpital à Aubagne." [...] Alors, je dis : "Ben, votre mère, vous n'avez qu'à la voir... Allez la voir dans les heures ou vous êtes autorisé à sortir, point barre." Parce que sinon on n'en finit plus. Quand il s'agit du travail, c'est peut-être plus... Enfin bon... Je veux dire, le travail, ils ont tellement de difficultés à trouver... La population qu'on côtoie a tellement de mal à trouver un travail que, quand elle en trouve un, on veut essayer. Mais bon, là, pour des problèmes de relations intrafamiliales, je pense qu'il peut, enfin bon, à moins que la mère soit vraiment mourante ou des trucs comme ça, je pense qu'il peut s'arranger autrement » (CIP, entretien $\left.n^{\circ} 8\right)$.

On voit bien l'accueil différent que reçoivent les arguments de la part des personnes en charge de la surveillance. L'emploi est une raison suffisante pour déroger à la règle préalablement établie quand l'hospitalisation d'un proche ne l'est pas. L'argument de la santé reste lui aussi un élément fort qui permet de justifier une sortie en violation comme dans le cas de ce placé, sorti pendant le couvre-feu pour se rendre chez son dentiste. Cet argument peut cependant poser problème: puisque la notion d'urgence est, dans l'exemple qui suit, très difficile à traiter, un accord ne peut être trouvé et la demande est rejetée.

« Le dentiste, est-ce que c'est une urgence ? Ben, c'est pas une urgence, ça. Vous n'êtes pas autorisé, vous le savez, à sortir en dehors de vos horaires quand il n'y a pas d'urgence médicale... Mais on ne peut pas autoriser une sortie pour aller chez le dentiste !» (conversation enregistrée au pôle de surveillance électronique des Baumettes le 22 octobre 2009).

Les glissements entre les différentes «cités » sont d'autant plus fréquents dans le cadre du PSE que ce dispositif s'intègre à l'ensemble de la vie quotidienne des personnes qui portent le bracelet quand la surveillance se limite aux heures de travail des téléacteurs et s'intègre dans une relation contractuelle. Puisque les façons de faire sont multiples (même si largement limitées par le cadrage technologique), les téléacteurs conservent une certaine maîtrise sur leur activité dont les placés ne peuvent bénéficier. Ceci explique certainement le fait que ces derniers mobilisent en priorité l'argumentation reposant sur les grandeurs, à même de produire quelque inflexion dans le jugement du surveillant quand les téléacteurs peuvent, eux, développer des logiques de retraduction que nous allons maintenant détailler. La capacité à ouvrir la négociation et à "réchauffer " les jugements dépend donc fortement du degré de liberté - relatif - du surveillé.

\section{Retraduction et contre-enquête}

Dans les situations de "contradiction" (Soler et Trompette, 2010) dont il va ici être question, le réel est réinterprété par le surveillé pour maintenir ou recréer une adéquation entre le comportement saisi et la règle. Ce faisant, il rend conforme un comportement qui, à première vue, semblait ne pas l'être. Il s'agit effectivement d'un phénomène de traduction (Callon, 1986), dans le sens où le surveillé redéfinit les éléments 
à prendre en compte en vue de produire une (re)qualification de la situation, concurrente ou opposée à celle du surveillant. Il déploie pour ce faire des processus comparables à ceux des surveillants chargés de mener l'enquête puisqu'il mène une " contre-enquête " pour les besoins de laquelle il mobilise les prises (Bessy et Chateauraynaud, 1993, 1995) et indices disponibles et leur attribue une signification nouvelle. À la traduction opérée par les dispositifs de capture - qui simplifient les situations -, les acteurs vont opposer une nouvelle traduction et réintroduire de la complexité au sein de ces épreuves nouvelles. Les pièces à conviction objectives que fournit la machine se transforment alors en moyen d'exhiber sa conformité.

« Ça, c'est un truc concret, c'est-à-dire, si toute la semaine ça va et que, le vendredi, tu passes de six appels par heure à quatre appels par heure, et que c'est tous les vendredis. Parce que tu vois que, en plus, je ne sais pas, à 80 \%, t'as un motif... "Ne répond pas", là tu peux dire au client : "Ben voilà, on a une baisse de productivité le vendredi... Ben parce que... Peut-être les gens sont en RTT, donc on a moins de monde au téléphone, forcément en volume, on fait moins de chiffre." »(Divy, téléacteur).

Divy combine ici différents éléments de la situation auxquels il attribue une signification particulière, et donc un rôle à jouer dans le processus de justification. Il requalifie les éléments de la situation : le vendredi n'est plus un jour de la semaine comme un autre, le taux de «non-réponse ", récurrent, permet de le requalifier. Le vendredi devient alors un " jour de RTT », au cours duquel il est logique que les résultats soient moins bons que le reste de la semaine puisque les personnes ne sont pas chez elles pour répondre au téléphone. Selon cette même logique, Mathieu combine différents indices pour prouver la piètre qualité des fichiers.

"Quand il n'y a pas de rendez-vous, tu vois, et que ça ne sort pas sur aucune des équipes, eh ben, ils ne vont quand même pas aller jeter la pierre aux fichiers, tu vois, ils jettent quand même la pierre sur les TA, c'est-à-dire que ce n'est jamais, jamais, jamais la faute des fichiers, jamais et, ça, je ne comprends pas, quoi. Tu vois, c'est un truc qui me révolte un peu, tu vois. Non, mais c'est vrai, tu vois, ce n'est c'est jamais la faute des fichiers, c'est toujours la faute des TA et, pourtant, ils savent très bien. [...] En 10 minutes, tu vois que t'as un fichier qui est pourri, quoi. Si en 20 appels que t'as passés, on t'a dit 15 fois ou 17 fois "On m'a déjà appelé 10 fois en une semaine ou en un mois, c'est du harcèlement, arrêtez", tu te fais jeter comme un malpropre, c'est que le fichier n'est pas bon, quoi ” (Mathieu, téléacteur).

Ce travail de traduction passe par une expertise des statistiques, des chiffres et de leur signification. Le sens est produit par la combinaison de différents éléments stockés par le dispositif. Divy et Mathieu mobilisent ce dernier pour éclairer leur pratique et celle de leurs collègues d'un jour nouveau. Moyennant le travail des chiffres, la responsabilité est transférée vers des éléments extérieurs sur lesquels les téléacteurs ne peuvent avoir de prise.

Les surveillés font également valoir les contraintes pratiques dans lesquelles ils se trouvent et qui cadrent leur activité. Cette réalité, faite d'imprévus et d'adaptations aux contingences locales, se heurte fréquemment à la rigidité des dispositifs de contrôle avec leurs programmes d'action fixes et stricts et n'est généralement pas prise en compte dans les modes d'évaluation (Jaeger, 2002). Il s'agit alors de trouver un compromis et de montrer au surveillant que dans la situation donnée agir de cette façon est la meilleure façon de faire, la " plus conforme », toute chose égale par ailleurs. 
32 En réchauffant des jugements que la technique tend fréquemment à refroidir, les surveillés agissent ici sur la réalité telle qu'il convient de la penser et de la définir. La déviance n'est plus opérante puisqu'elle est jugée depuis un point de vue sur la situation qui ne correspond pas à la réalité. Luc Boltanski distingue le "monde ", "considéré comme étant - pour reprendre la formule de Wittgenstein - «tout ce qui arrive » et la "réalité", "ce qui dans le monde a été pris en charge, de façon réflexive, par les épreuves (de réalité)» (2008, p. 19). Les surveillés offrent à voir du "monde » une autre " réalité », où leur comportement ne saurait être jugé négativement eu égard à ce qu'il convient de prendre en compte et de considérer comme pertinent pour le qualifier.

\section{Discussion}

\section{Objectivité et neutralité}

Nous assistons dans de nombreux contextes à la volonté grandissante de s'assurer d'une saisie - et des contrôles associés - toujours plus fine et précise des comportements, et on confère aisément à la technique une objectivité et une neutralité certaines. Si nous saisissons bien la puissance de ces « technologies invisibles » (Berry, 1983) et observons la façon dont «l'automatisation dépersonnalise les sentences»(Dujarier, 2010, p. 150), l'agencement sociotechnique dont nous faisons ici état laisse pourtant in fine à l'humain le soin de procéder aux arbitrages concernant la légitimité et de mettre en place les éventuelles actions correctives. Si son jugement est largement équipé par la technique, le système ne peut clore seul l'enquête et s'en remet au surveillant qui reste celui qui tranche et qui juge de la conformité ou statue sur la déviance.

Loin de la rigidité des programmes informatiques, la conformité apparaît, au terme de notre développement, comme un objet difficile à saisir et à définir, et ce malgré les nombreux équipements technologiques chargés de soutenir les jugements et de réduire l'incertitude. Bien loin d'une vision substantialiste de la conformité, celle-ci apparaît alors comme le fruit d'un processus complexe et polymorphe de qualification, le résultat de constantes négociations fortement encastrées dans des relations sociales. Autour de sa définition et des jugements auxquels elle donne lieu, « réapparaît tout un cheminement fait d'épreuves, de contradictions, de désaccords et de controverses » (Soler et Trompette, 2010 , p. 324). Le fait que la conformité soit un « objet complexe » (Mol, 2002) permet par ailleurs aux surveillants de ne pas apparaître, au sein du dispositif, comme de simples agents d'enregistrement. Ils doivent au contraire faire preuve de "discernement", pondérer les différents indices à leur disposition, complétant en définitive le travail de la machine. La surveillance est imparfaite, surveillants et surveillés en sont conscients ; elle doit pourtant, pour fonctionner, conserver l'aspect d'un dispositif implacable auquel rien n'échappe.

L'exercice objectif, apolitique, du pouvoir et de la surveillance apparaît une nouvelle fois comme une illusion, et ce malgré l'intervention objectivante de la machine. On comprend bien la part d'arbitraire que contient ce dispositif dans la mesure où il est :

« impossible d'en prendre la mesure en le référant à un format préétabli assurant à son exercice une certaine constance et, par-là, de mettre l'accent sur les difficultés auxquelles sont confrontés ceux qui le subissent pour former à son égard des attentes légitimes » (Boltanski, 2009, p. 16). 
Nous constatons de fait que ce dispositif croise « un enjeu purement descriptif, appuyé par une pratique prétendument neutre de récolte de données, et un enjeu normatif » (Rouvroy et Berns, 2009, p. 8). Enjeux et présupposés normatifs et moraux que renferme la technique (Akrich, 1987), qui tendent à disparaitre ou à être invisibilisés dans et par l'usage et son mode de fonctionnement. Il semble pourtant que les logiques de négociation ici décrites, en rendant complexe l'objet sur lequel repose le principe de sanction, entraînent une première forme de remise en cause de l'ordre (au sens d'ordre social) imposé - en partie au moins, au moyen de la technique. Ce dernier ne va plus de soi et peut alors potentiellement être soumis à la critique, à la résistance. A contrario, les évolutions techniques et organisationnelles récentes peuvent être lues comme allant dans le sens d'une rigidification du système. Les nouveaux bracelets électroniques, par exemple, qui imposent un traitement systématique de toutes les alarmes, interdisent que les rapports d'incidents soient faits à la discrétion du surveillant. Les écarts, mêmes mineurs, donnent lieu à un signalement et on se rapproche peu à peu des formes de « gouvernement statistique » et de "profilage » (Rouvroy et Berns, op. cit.) avec lesquels il n'est plus guère possible de négocier.

\section{La critique et son objet}

Si ces négociations et contournements de la surveillance représentent une opportunité critique, il convient d'analyser plus en détail son objet et le rôle que joue la technique dans la critique éventuelle à laquelle donne lieu la « dispute technologique ». À ce titre, il parait pertinent de distinguer ici critique procédurale et critique en finalité.

La critique procédurale porte sur les modalités concrètes d'exercice de la surveillance. C'est une critique localisée : B critique $C$ ou ce qu'il est en train de faire. La critique en finalité porte quant à elle sur les enjeux et les principes que sous-tend la situation de surveillance. La critique est alors globale : B remet en question A.

Schéma 4 : critique procédurale et critique en finalité
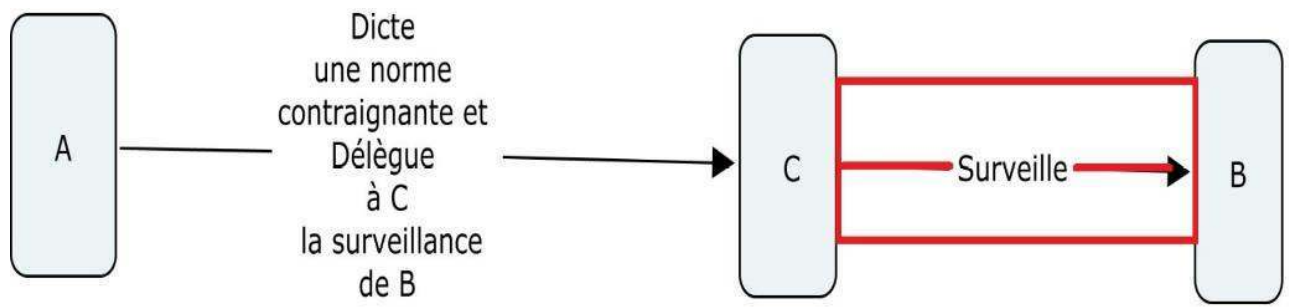

La surveillance électronique peut être mobilisée comme un levier critique, dans une forme a posteriori de mise à l'épreuve de l'objet. Bien souvent au cours de l'enquête, les prisonniers ont fait clairement allusion à des logiques de déshumanisation, voire d'animalisation («J'ai l'impression d'être un chien en laisse/attaché »; « D'abord, il y a le côté humiliant »). Les salariés des centres d'appels évoquent eux aussi très régulièrement les problèmes de stress et de pression professionnelle auxquels ils sont quotidiennement confrontés et remettent en cause les conditions difficiles d'exercice d'une activité jugée négativement. Pourtant, l'élaboration de ce type de discours nécessite une prise de distance réflexive sur la situation qui reste relativement exceptionnelle tant la pratique est saturée d'autres occasions de préoccupation. En situation, ce sont en effet les façons de faire bien plus que les principes sous-jacents à l'activité qui sont contournés, discutés, négociés ou remis en cause dans le cadre d'une critique procédurale ou fonctionnelle, 
largement ancrée dans les dimensions pratiques de l'exercice du contrôle ${ }^{7}$; les surveillés mettent en place des "tactiques" pour jouer avec le système, mais n'élaborent à son encontre que peu de "stratégies" de résistance (de Certeau, 1990). Si les exemples mobilisés au cours de cet exposé laissent à voir des situations où la règle est réaménagée par le surveillé (et par le surveillant), nous constatons aussi que la finalité, quant à elle, n'est pour ainsi dire jamais affectée. Dans de telles conditions, quelle est donc la portée critique de la "dispute technologique»? La technologie n'assure-t-elle pas, outre ses fonctions premières de saisie et d'alarme, un rôle plus symbolique de cristallisateur des critiques qui ne sont plus adressées aux principes fondamentaux orientant l'action, mais à la réalité concrète et matérielle de leur mise en œuvre uniquement ? Comme le note MA. Dujarier, «la mesure et le jugement automatisés ne sont pas le pouvoir, mais l'outil d'un rapport de pouvoir, sa justification en même temps que son camouflage » (2010, p. 151). Puisque c'est en définitive avec les machines ou à leur propos que les individus négocient leur surveillance, elles deviennent logiquement le point focal de revendication quand elles ne font bien sûr qu'équiper des programmes dont les enjeux dépassent ces seules considérations matérielles, mais concernent les modes de production, d'organisation et de contenu du travail, de gouvernement des corps ou de "vivre ensemble ». Offrant au surveillé l'occasion de l'expression d'une critique, ces dispositifs contribuent également peut-être, dans une certaine mesure, à rendre tolérable une situation qui ne l'est que peu mais dont il est par ailleurs impossible de s'extraire.

AGRE P. E., 1994, « Surveillance and Capture: Two Models of Privacy », The Information Society, vol. 10, n 2, pp. 101-127.

AKRICH M., 1990, « De la sociologie des techniques à une sociologie des usages : l'impossible intégration du magnétoscope dans les réseaux câblés de première génération ", Techniques \& Culture, $\mathrm{n}^{\circ} 16$, pp. 83-110.

ALLARIA C., 2014, «Le Placement sous surveillance électronique : espace et visibilité du châtiment virtuel », Champ pénal/Penal Field, vol. XI.

BERRY M., 1983, Une technologie invisible - L'impact des instruments de gestion sur l'évolution des systèmes humains, Centre de recherche en gestion, École polytechnique.

BESSY C. et CHATEAURAYNAUD, F., 1993, «Les Ressorts de l'expertise : épreuves d'authenticité et engagement des corps ", in B. CONEIN, N. DODIER et L. THÉVENOT, Les Objets dans l'action : De la maison au laboratoire, Paris, Éditions de l'EHESS, coll. « Raisons pratiques », n 4, pp. 141-164.

BESSY C. et CHATEAURAYNAUD, F., 1995, Experts et faussaires : Pour une sociologie de la perception, Paris, Métailié.

BOLTANSKI L. et THÉVENOT, L., 1991, De la justification : Les Économies de la grandeur, Paris, Gallimard, coll. « NRF Essais ».

BOLTANSKI L., 2009, De la critique : Précis de sociologie de l'émancipation, Paris, Gallimard, coll. « NRF Essais ».

BOLTANSKI L., 2008, «Institutions et critique sociale : Une approche pragmatique de la domination », Tracés, hors-série $n^{\circ} 8$.

BUSCATO M., 2002, « Les Centres d'appels, usines modernes ? Les rationalisations paradoxales de la relation téléphonique », Sociologie du travail, vol. 44, n 1, pp. 99-117.

CALLON M., 1986, « Éléments pour une sociologie de la traduction. La domestication des coquilles Saint-Jacques dans la Baie de Saint-Brieuc », L'Année sociologique, $\mathrm{n}^{\circ} 36$. 
CERTEAU M. (de), 1990, L'Invention du quotidien : Arts de faire, t. I, Paris, Gallimard, coll. « Folio Essais ».

CHAULET J., 2007, La Confiance médiatisée : La Confiance et sa gestion au sein des communications médiatisées, thèse de doctorat de l'université Toulouse Le Mirail.

CHAULET J., 2006, « Parler et faire parler : travail en centre d'appels et dispositif de surveillance équipée ", Terrain \& Travaux, $n^{\circ} 11$, pp. 36-60.

CHAUVENET A., BENGUIGUI G. et ORLIC F., 1993, «Les Surveillants de prison : le prix de la sécurité ", Revue française de sociologie, vol. 34, n 3, pp. 345-366.

CROZIER M. et FRIEDBERG E., 1992, L'Acteur et le système : Les Contraintes de l'action collective, Paris, Le Seuil, coll. « Points Essais ».

DEVRESSE M.-S., 2013, « Être placé sous surveillance électronique : Une forme originale de "peine situationnelle" ? ", Déviance et Société, vol. 37, n³.

DEVRESSE M.-S., 2007, « Innovation pénale et surveillance électronique : quelques réflexions sur une base empirique ", Champ pénal/Penal Field [En ligne], Séminaire Innovations Pénales, mis en ligne le 29 septembre 2007, consulté le 08 octobre 2016.

DODIER N., 2005, «L'Espace et le mouvement du sens critique », Annales. Histoire, Sciences Sociales, Paris, Éditions de l'EHESS, vol. 1, 60e année, pp. 7-31.

DUJARIER M.-A., 2010, « L'Automatisation du jugement sur le travail. Mesurer n'est pas évaluer », Cahiers internationaux de sociologie, vol. 1, $\mathrm{n}^{\circ}$ 128-129.

EMA A. et FUJIGAKI Y., 2011, « How far can child surveillance go?: Assessing the parental perceptions of an RFID child monitoring system in Japan ", Surveillance \& Society, vol. $9 \mathrm{n}^{\circ} 1 / 2$, pp. 132-148.

FOUCAULT M., 2001 [1974], « La Vérité et les formes juridiques », in Dits et Écrits, tome II (texte n -139), Paris, Quarto.

FOUCAULT M., 1993 [1975], Surveiller et punir : Naissance de la prison, Paris, Gallimard, coll. « Tel ». GREEN N., 2002, «Qui surveille qui ? Contrôler et rendre des comptes dans les relations de téléphonie mobile », Réseaux, vol. 2, n 112-113, pp. 250-273.

JAEGER C., 2002, « L'Impossible Évaluation du travail des téléopérateurs : le cas de deux centres d'appel », Réseaux, vol. 4, n 114, pp. 51-90.

KALUSZYNSKI M. et FROMENT J.-C., 2003, « Sécurité et nouvelles technologies. Évaluation comparée dans cinq pays européens (Belgique, Espagne, France, Grande-Bretagne, Suisse) des processus de recours au placement sous surveillance électronique », Grenoble, CERAT-IEP.

KOBELINSKY C., 2008, «Les Situations de retour. Restituer sa recherche à ses enquêtés ", in D. FASSIN et A. BENSA (dir.), Les Politiques de l'enquête : Épreuves ethnographiques, Paris, La Découverte. LANDREVILLE P., 1987, « Surveiller et prévenir. L'assignation à domicile sous surveillance électronique », Déviance et Société, vol. 11, n³. pp. 251-269.

LÉVY R. et PITOUN A., 2004, « L'Expérimentation du placement sous surveillance électronique en France et ses enseignements (2001-2004) », Déviance et Société, n 4, vol. 28, pp. 411-437.

LIANOS M., 2003, « Le Contrôle social après Foucault », Surveillance \& Society, vol. 1, nº 3.

MOL A., 2003, The Body Multiple : Ontology in Medical Practice. Durham, Duke University Press. 
RAZAC 0., 2013, « La Matérialité de la surveillance électronique », Déviance et société, vol. 37, n 3, pp. 389-403.

RAZAC 0., 2010, « Le Placement sous surveillance électronique mobile : un nouveau modèle pénal ? ", rapport CIRAP, Paris, École nationale d'administration pénitentiaire.

ROONEY T., 2010, « How do surveillance technologies alter a child's experience of trust, risk and responsibility? », Surveillance \& Society, vol. 7, n 3/4, pp. 344-355.

ROUVROY A. et BERNS T., 2009, « Le Corps statistique », La Pensée et les Hommes, n 74, pp. 173-194.

SCHWEITZER L., 2003, « TIC et contrôle social : regards sur le travail dans les centres d'appels », Terminal, $n^{\circ} 89$, pp. 101-110.

SOLER J. et TROMPETTE P., 2010, « Une technologie pour la santé : traces et expertises. Chercheurs, familles et médecins autour de la définition des crises d'épilepsie ", Revue d'anthropologie des connaissances, vol. 4, $\mathrm{n}^{\circ}$ 2, pp. 323-357.

THÉVENOT L., 2006, L'Action au pluriel : Sociologie des régimes d'engagement, Paris, La Découverte.

\section{NOTES}

1. On peut citer, à titre d'exemple, la surveillance des enfants (Ema et Fujigaki, 2011 ; Rooney, 2010), des salariés (Buscatto, 2002; Chaulet, 2006; Schweitzer, 2003) ou des prisonniers (Landreville, 1987 ; Kaluszynski et Froment, 2003 ; Lévy et Pitoun, 2004 ; Razac, 2010, 2013 ; Devresse, 2007, 2013 ; Allaria, 2014).

2. Nous en voulons pour preuve la relative stabilité - voire extension - de l'un et l'autre de nos objets.

3. Le terme "placé» est utilisé pour désigner les personnes placées sous surveillance électronique. Sur le terrain, les surveillants, les conseillers pénitentiaires d'insertion et de probation et les magistrats peuvent être amenés à employer ce terme par raccourci de langage.

4. Ces variations s'expliquent techniquement par le rayon de captation du dispositif et la présence d'éventuels obstacles.

5. Outre le nombre de ventes par heure à réaliser, les téléopérateurs se voient également imposé un nombre de «contacts» et de "contacts argumentés». Si le premier indice est le plus important, puisqu'il est l'élément qui produit de la valeur commerciale, le second est également surveillé et pris en compte.

6. Elles se distinguent en ce sens des formes de "gouvernementalité statistique » que décrivent A. Rouvroy et T. Berns dans le sens où le surveillé conserve ici la possibilité de « faire valoir son désaccord avec une loi qu'il estime injuste, ou les raisons personnelles et contextuelles qui justifient ou excusent son infraction ou doivent lui faire bénéficier de l'impact de circonstances atténuantes » (2009).

7. En ce sens, notre distinction se rapproche de la dialectique entre «épreuve " et " pouvoir» que propose N. Dodier lorsqu'il examine la notion de critique; les épreuves renvoient à la dimension procédurale et concrète de "clôture des justifications ", le pouvoir aux finalités et aux «biens en soi » sur lesquels elle s'appuie (Dodier, 2005). 


\section{ABSTRACTS}

This article seeks to question diversions of and negotiations around ICT-mediated surveillance, as well as their eventual critical scope. Our analysis relies on ethnographic data collected during two field studies - one dealing with surveillance of call centre employees, the other one with prisoners' electronic tags. We examine the ways in which those under surveillance play with the normative gaps of these devices to demystify surveillance equipment, or even manipulate it to distort data collection. These sociotechnical surveillance systems do not completely reduce uncertainty. This uncertainty is mobilized by those supervised to bypass the system or produce conformity. Practices such as these seem to have a limited critical scope because they deal with the concrete conditions of surveillance rather than with the philosophy on which the system are built.

Notre article entend questionner les contournements et négociations auxquels les systèmes de surveillance équipés par les TIC donnent lieu, ainsi que leur éventuelle portée critique. Nous mobilisons ici les données ethnographiques recueillies sur deux terrains d'enquête : un centre d'appels téléphoniques et un pôle de surveillance électronique des condamnés. Nous examinons la façon dont les individus surveillés « jouent » avec les interstices normatifs de ces dispositifs en démystifiant le matériel de surveillance, voire en le manipulant de façon à détourner les traces numériques. Ces systèmes sociotechniques de surveillance ne permettent pas de réduire totalement l'incertitude concernant la situation du surveillé et son activité. Cette incertitude est mobilisée par les surveillés pour contourner le dispositif ou produire de la conformité. Enfin, la discussion traitera de la portée critique limitée que ces pratiques de négociation semblent revêtir. En effet, ce sont sur les conditions concrètes de la surveillance que porte la négociation bien plus que sur les principes sur lesquels, elles reposent.

El presente artículo busca cuestionar las formas de contornear y de negociar existentes en los sistemas de vigilancia basados en las tecnologías de la información y de la comunicación; además de dar a conocer el alcance crítico de las mismas. Los datos etnográficos utilizados fueron recogidos en dos ámbitos: una centralita telefónica y un centro de vigilancia electrónica de una cárcel. Se analiza la manera en que los individuos vigilados "juegan" con los intersticios normativos de estos dispositivos de control, desmitificando el material de vigilancia, llegando incluso a manipulaciones para desviar las huellas digitales. Esos sistemas socio-técnicos de vigilancia no permiten reducir totalmente la incertidumbre relativa a la situación del supervisado y de su actividad; incertidumbre que es utilizada por los vigilados para soslayar el dispositivo o para crear conformidad. Finalmente, la discusión analiza el alcance crítico limitado que estas prácticas de negociación parecen representar: en efecto, la negociación tiene más relación con las condiciones concretas de la vigilancia que con los principios sobre las que dichas condiciones se establecen. 
INDEX

Mots-clés: surveillance électronique, critique, négociation, bracelet électronique, centre d'appels

Palabras claves: vigilancia electrónica, critica, negociación, brazalete de geo-posicionamiento, centralita telefónica.

Keywords: electronic monitoring, surveillance, negotiation, electronic tag, call centre

\section{AUTHORS}

\section{JOHANN CHAULET}

Johann Chaulet est sociologue, chargé de recherches au CNRS au sein du LISST de l'université Toulouse Jean Jaurès. Il est spécialisé dans les usages relationnels des technologies de l'information et de la communication. Il est le codirecteur de ComUniTIC (communauté des universitaires autour des usages des TIC).

\section{CAMILLE ALLARIA}

Camille Allaria est sociologue, chercheuse associée au LAMES à Aix-en-Provence, et elle travaille en collaboration avec l'Observatoire régional de la délinquance et des contextes sociaux (ORDCS). Elle développe aujourd'hui des travaux principalement axés autour de la sociologie de la déviance et des institutions. 